\title{
CENTERS FOR GENERALIZED QUINTIC POLYNOMIAL DIFFERENTIAL SYSTEMS
}

\author{
JAUME GINÉ ${ }^{1}$, JAUME LLIBRE $^{2}$ AND CLAUDIA VALLS ${ }^{3}$
}

\begin{abstract}
We classify the centers of the polynomial differential systems in $\mathbb{R}^{2}$ of degree $d \geq 5$ odd that in complex notation writes as

$\dot{z}=i z+(z \bar{z})^{\frac{d-5}{2}}\left(A z^{5}+B z^{4} \bar{z}+C z^{3} \bar{z}^{2}+D z^{2} \bar{z}^{3}+E z \bar{z}^{4}+F \bar{z}^{5}\right)$, where $A, B, C, D, E, F \in \mathbb{C}$ and either $A=\operatorname{Re}(D)=0$, or $A=$ $\operatorname{Im}(D)=0$, or $\operatorname{Re}(A)=D=0$, or $\operatorname{Im}(A)=D=0$.
\end{abstract}

\section{Introduction And STATEMENT of the MAin RESUlts}

In the qualitative theory of real planar polynomial differential systems one of the main problems is the center-focus problem, i.e. the problem of distinguishing between a center and a focus. For singular points whose linear part has a pair of pure imaginary eigenvalues this problem is equivalent to the existence of an analytic first integral defined in a neighborhood of the singular point, see for more details the articles $[24,25]$ and $[2,13,14]$.

A singular point is a center if there exists a neighborhood of it such that all the orbits in this neighborhood are periodic except the singular point, and a singular point is a focus if there is a neighborhood of it such that all the orbits in this neighborhood spiral either in forward or in backward time to the singular point.

We study the center-focus problem for a class of polynomial differential systems which generalize the class of linear polynomial differential systems with homogeneous polynomial nonlinearities of degree 5 . The characterization of the centers of the polynomial differential systems started with the classes of all the quadratic polynomial differential systems and the linear polynomial systems with homogeneous polynomial nonlinearities of degree 3, see for instance [1, 27, 28, 29, 30]. Unfortunately in the present we are very far from having the classification of all the centers of the cubic polynomial differential systems. But some

2010 Mathematics Subject Classification. Primary 34C05. Secondary 37C10.

Key words and phrases. nilpotent center, degenerate center, Lyapunov constants, Bautin method. 
subclasses of cubic polynomial differential systems with centers are studied, see for instance the papers $[31,32]$ and references quoted there. The centers of linear polynomial differential systems with homogeneous polynomial nonlinearities of degree $k>3$ are not classified, but there are many partial results for $k=4,5,6,7,9$ see $[3,4,12,20,21,22,23]$. In general the huge amount of computations which are necessary for obtaining the complete classification becomes the center problem intractable computationally, see for instance [16] and references quoted there.

In this paper we work with the real planar polynomial differential systems that has a singular point at the origin with eigenvalues $\pm i$ and that in complex can be written as

(1) $\dot{z}=i z+(z \bar{z})^{\frac{d-5}{2}}\left(A z^{5}+B z^{4} \bar{z}+C z^{3} \bar{z}^{2}+D z^{2} \bar{z}^{3}+E z \bar{z}^{4}+F \bar{z}^{5}\right)$,

where $z=x+i y, d \geq 5$ is an arbitrary odd integer and $A, B, C, D, E, F \in$ $\mathbb{C}$ satisfying one of the following four conditions:

$$
\begin{aligned}
& \text { (c.1) } A=\operatorname{Re}(D)=0, \\
& \text { (c.2) } A=\operatorname{Im}(D)=0, \\
& \text { (c.3) } \operatorname{Re}(A)=D=0, \\
& \text { (c.4) } \operatorname{Im}(A)=D=0 .
\end{aligned}
$$

These systems contens as a particular case the results of the paper [21], where the authors characterize the centers of the system (1) with $A=D=0$.

The polynomial differential systems (1) when $d=5$ coincide with the class of quintic polynomial differential systems of the form a linear center plus homogeneous polynomial nonlinearities of degree 5. Therefore the polynomial differential systems (1) of odd degree $d>5$ generalizes the class of linear polynomial differential systems with quintic homogeneous polynomial nonlinearities.

The main result of this paper is the characterization of the centers for the polynomial differential systems (1) under the assumptions (c.1)-(c.4). We present the classification of these centers in a different theorem for each of the four classes.

Theorem 1. The polynomial differential systems (1) satisfying conditions (c.1) have a center at the origin if one of the following conditions hold.

(a) $\operatorname{Re}(C)=\operatorname{Im}(D)=\operatorname{Re}(\bar{B} E \bar{F})=\operatorname{Re}\left(B^{2} E\right)=\operatorname{Im}\left(B E^{2} \bar{F}\right)=$ $\operatorname{Im}\left(B^{2} E \bar{F}\right)=\operatorname{Im}\left(B^{3} F\right)=\operatorname{Re}\left(E^{3} \bar{F}^{2}\right)=0$,

(b) $\operatorname{Re}(B)=\operatorname{Re}(C)=F=3 B+\bar{D}=0$,

(c) $\operatorname{Re}(B)=\operatorname{Re}(C)=\operatorname{Re}(E)=\operatorname{Re}(F)=0$, 
(d) $\operatorname{Re}(C)=E=2 B+\bar{D}=0$.

The proof of Theorem 1 is given in section 3 .

Theorem 2. The polynomial differential systems (1) satisfying conditions (c.2) have a center at the origin if one of the following conditions hold.

(a) $\operatorname{Re}(C)=\operatorname{Im}(D)=\operatorname{Re}(\bar{B} E \bar{F})=\operatorname{Re}\left(B^{2} E\right)=\operatorname{Im}\left(B E^{2} \bar{F}\right)=$ $\operatorname{Im}\left(B^{2} E \bar{F}\right)=\operatorname{Im}\left(B^{3} F\right)=\operatorname{Re}\left(E^{3} \bar{F}^{2}\right)=0$,

(b) $\operatorname{Re}(B)=\operatorname{Re}(C)=F=3 B+\bar{D}=0$,

(c) $\operatorname{Im}(B)=\operatorname{Re}(C)=\operatorname{Re}(E)=\operatorname{Im}(F)=0$,

(d) $\operatorname{Re}(C)=E=2 B+\bar{D}=0$.

We note that the change of variables $(8)$ with $\xi=\left(\left(a_{8} / a_{7}\right) e^{-i \pi / 4}\right)^{1 / 4}$ transforms condition (c.2) into condition (c.1). Therefore Theorem 2 will not be proved.

Theorem 3. The polynomial differential systems (1) satisfying conditions (c.3) have a center at the origin if one of the following conditions hold.

(a) $\operatorname{Re}(C)=\operatorname{Im}(D)=\operatorname{Re}(\bar{B} E \bar{F})=\operatorname{Re}\left(B^{2} E\right)=\operatorname{Im}\left(B E^{2} \bar{F}\right)=$ $\operatorname{Im}\left(B^{2} E \bar{F}\right)=\operatorname{Im}\left(B^{3} F\right)=\operatorname{Re}\left(E^{3} \bar{F}^{2}\right)=0$,

(b) $\operatorname{Re}(C)=B=5 \bar{A}+E=0$,

(c) $\operatorname{Re}(C)=A-3 \bar{E}=F=0$,

(d) $C=F=\operatorname{Re}(E)=\operatorname{Re}(B)-\operatorname{Im}(B)=7 A+E=49 \operatorname{Im}(B)^{2}-$ $8 \operatorname{Im}(E)^{2}=0$ and $d=5$,

(e) $C=F=\operatorname{Re}(E)=\operatorname{Re}(B)+\operatorname{Im}(B)=7 A+E=49 \operatorname{Im}(B)^{2}-$ $8 \operatorname{Im}(E)^{2}=0$ and $d=5$,

(f) $C=F=\operatorname{Re}(E)=3 A+E=9|B|^{2}-16|E|^{2}=0$ and $d=5$,

(g) $B=C=3 A-5 \bar{E}=16|E|^{2}-9|F|^{2}=0, F=|F| e^{i \psi}$ with $\psi=\pi / 4+k \pi, k \in \mathbb{Z}$ and $d=5$,

(h) $\operatorname{Re}(B)=\operatorname{Re}(C)=\operatorname{Re}(E)=\operatorname{Re}(F)=0$,

(i) $\operatorname{Re}(C)=A-C=E=B+\bar{F}=|C|^{2}-|F|^{2}=0$ and $d=5$,

(j) $\operatorname{Re}(C)=A+C=E=B-\bar{F}=|C|^{2}-|F|^{2}=0$ and $d=5$,

(k) $\operatorname{Re}(C)=\operatorname{Re}(E)$, conditions (36) and $d=5$,

(l) $C=B+\bar{F}=\operatorname{Re}(E)=A+E=4|E|^{2}-|F|^{2}=0$ and $d=5$,

(m) $C=B-\bar{F}=\operatorname{Re}(E)=A+E=4|E|^{2}-|F|^{2}=0$ and $d=5$,

(n) $\operatorname{Im}(B)=\operatorname{Re}(C)=\operatorname{Im}(E)=\operatorname{Im}(F)=0$.

The proof of Theorem 3 is given in section 4 . Note that the statement (a) of Theorem 3 coincides with the statement (a) of Theorem 1, and consequently it will not be proved. 
Theorem 4. The polynomial differential systems (1) satisfying conditions (c.4) have a center at the origin if one of the following conditions hold.

(a) $\operatorname{Re}(C)=\operatorname{Im}(D)=\operatorname{Re}(\bar{B} E \bar{F})=\operatorname{Re}\left(B^{2} E\right)=\operatorname{Im}\left(B E^{2} \bar{F}\right)=$ $\operatorname{Im}\left(B^{2} E \bar{F}\right)=\operatorname{Im}\left(B^{3} F\right)=\operatorname{Re}\left(E^{3} \bar{F}^{2}\right)=0$,

(b) $\operatorname{Re}(C)=B=5 \bar{A}+E=0$,

(c) $\operatorname{Re}(C)=A-3 \bar{E}=F=0$,

(d) $C=F=\operatorname{Im}(B)=\operatorname{Im}(E)=7 B+4 E=7 A-E=0$ and $d=5$,

(e) $C=F=\operatorname{Im}(B)=\operatorname{Im}(E)=7 B-4 E=7 A-E=0$ and $d=5$,

(f) $C=F=\operatorname{Im}(E)=3 A+E=9|B|^{2}-16 \operatorname{Re}(E)^{2}=0$ and $d=5$,

(g) $B=C=3 A-5 \bar{E}=16|E|^{2}-9|F|^{2}=0 F=|F| e^{i \psi}$ with $\psi=k \pi / 2, k \in \mathbb{Z}$ and $d=5$,

(h) $E=\operatorname{Re}(C)=\operatorname{Re}(A)-\operatorname{Im}(C)=B+i \bar{F}=|C|^{2}-|F|^{2}=0$ and $d=5$,

(i) $E=\operatorname{Re}(C)=\operatorname{Re}(A)+\operatorname{Im}(C)=|C|^{2}-|F|^{2}=B-i \bar{F}=0$ and $d=5$,

(j) $\operatorname{Re}(C)=\operatorname{Im}(E)=\operatorname{Im}(C)^{2}-|F|^{2}=|B|^{2}-4 \operatorname{Re}(E)^{2}=a_{1}+a_{9}=$ $a_{3} a_{11}-a_{4} a_{12}=2 a_{6} a_{9}-a_{4} a_{11}-a_{3} a_{12}=a_{4} a_{6}-2 a_{9} a_{11}=a_{3} a_{6}-$ $2 a_{9} a_{12}=a_{4}^{2} a_{11}-4 a_{9}^{2} a_{11}+a_{3} a_{4} a_{12}=0$ and $d=5$,

(k) $C=\operatorname{Im}(E)=B+i \bar{F}=A-E=4|E|^{2}-|F|^{2}=0$ and $d=5$,

(l) $C=\operatorname{Im}(E)=B-i \bar{F}=A-E=4|E|^{2}-|F|^{2}=0$ and $d=5$,

(m) $\operatorname{Re}(C)=\operatorname{Im}(E)=\operatorname{Re}(F)-\operatorname{Im}(F)=\operatorname{Re}(B)-\operatorname{Im}(B)=0$,

(n) $\operatorname{Re}(C)=\operatorname{Im}(E)=\operatorname{Re}(F)+\operatorname{Im}(F)=\operatorname{Re}(B)+\operatorname{Im}(B)=0$.

We note that the change of variables (8) with $\xi=\left(\left(a_{2} / a_{1}\right) e^{i \pi / 2}\right)^{1 / 4}$ transforms condition (c.4) into condition (c.3). Hence Theorem 4 will not be proved.

\section{PRELIMINARY DEFinitions AND RESUlts}

There are very few results about the centers for classes of polynomial differential systems of arbitrary degree. The resolution of this problem implies the effective computation of the Poincaré-Liapunov constants. Indeed, setting

$$
\begin{aligned}
& A=a_{1}+i a_{2}, \quad B=a_{3}+i a_{4}, \quad C=a_{5}+i a_{6}, \\
& D=a_{7}+i a_{8}, \quad E=a_{9}+i a_{10}, \quad F=a_{11}+i a_{12},
\end{aligned}
$$

and writing (1) in polar coordinates, i.e., doing the change of variables $r^{2}=z \bar{z}$ and $\theta=\arctan (\operatorname{Im} z / \operatorname{Re} z)$, system (1) becomes

$$
\dot{r}=F(\theta) r^{d}, \quad \dot{\theta}=1+G(\theta) r^{d-1},
$$


where $F(\theta)$ and $G(\theta)$ are the homogeneous trigonometric polynomials

$$
\begin{aligned}
F(\theta)= & a_{5}+\left(a_{3}+a_{7}\right) \cos (2 \theta)+\left(a_{8}-a_{4}\right) \sin (2 \theta)+\left(a_{1}+a_{9}\right) \cos (4 \theta) \\
& +\left(a_{10}-a_{2}\right) \sin (4 \theta)+a_{11} \cos (6 \theta)+a_{12} \sin (6 \theta), \\
G(\theta)= & a_{6}+\left(a_{4}+a_{8}\right) \cos (2 \theta)+\left(a_{3}-a_{7}\right) \sin (2 \theta)+\left(a_{10}+a_{2}\right) \cos (4 \theta) \\
& +\left(a_{1}-a_{9}\right) \sin (4 \theta)+a_{12} \cos (6 \theta)-a_{11} \sin (6 \theta) .
\end{aligned}
$$

In order to determine the necessary conditions to have a center we propose the Poincaré series

$$
H(r, \theta)=\sum_{n=2}^{\infty} H_{n}(\theta) r^{n},
$$

where $H_{2}(\theta)=1 / 2$ and $H_{n}(\theta)$ are homogeneous trigonometric polynomials respect to $\theta$ of degree $n$. Imposing that this power series is a formal first integral of system (2) we obtain

$$
\dot{H}(r, \theta)=\sum_{k=2}^{\infty} V_{2 k} r^{2 k} \text {. }
$$

where $V_{2 k}$ are the Poincaré-Lyapunov constants that depend on the parameters of system (1). Indeed it is easy to see by the recursive equations that generate the $V_{2 k}$ that these $V_{2 k}$ are polynomials in the parameters of system (1), see [9]. As system (1) is polynomial, due to the Hilbert Basis theorem, the ideal $J=<V_{2}, V_{4}, \ldots>$ generated by the Poincaré-Liapunov constants is finitely generated, i.e. there exist $W_{1}, W_{2}, \ldots, W_{k}$ in $J$ such that $J=<W_{1}, W_{2}, \ldots, W_{k}>$. Such a set of generators is called a basis of $J$ and the conditions $W_{j}=0$ for $j=1, \ldots, k$ provide a finite set of necessary conditions to have a center. The set of coefficients for which all the Poincaré-Liapunov constants $V_{2 k}$ vanish is called the center variety of the family of polynomial differential systems and also it is an algebraic set.

In practice we determine a number of Poincaré-Liapunov constants thinking that inside these number there is the set of generators of the all Poincaré-Liapunov constants. From this set the much harder problem is decompose this algebraic set into its irreducible components. For simple cases this can be done by hand, see [3, 4, 15, 18, 19, 21]. However for more difficult systems the use of a computer algebra system is essential. The computational tool which we use is the routine minAssGTZ [8] of the computer algebra system SingulaR [11] which is based on the Gianni-Trager-Zacharias algorithm [10]. Since computations are too laborious they cannot be completed in the field of rational numbers. Therefore, we choose the approach based on making use of modular computations [26]. We have chosen the prime $p=32003$. To 
perform the rational reconstruction we used the MATHEMATICA code and the algorithm presented in [26]. The last step of this algorithm has not been verified because computations cannot be overcome. This step ensures that all the points of the center variety have been found. That is, we know that all the encountered points belong to the decomposition of the center variety but we do not know whether the given decomposition is complete. We remark that, nevertheless, it is practically sure that the given list is complete, see also [26]. Therefore, in the following we provide sufficient conditions to have a center, which are practically necessary.

From system (2) we can obtain the associated equation

$$
\frac{d r}{d \theta}=\frac{F(\theta) r^{d}}{1+G(\theta) r^{d-1}}
$$

It is clear that equation (4) is well defined in a sufficient small neighborhood of the origin. Hence if system (2) has a center at the origin, then equation (4) when $\dot{\theta}>0$ also has a center at the origin. The transformation $(r, \theta) \rightarrow(\rho, \theta)$ introduced by Cherkas [5] defined by

(5) $\rho=\frac{r^{d-1}}{1+G(\theta) r^{d-1}}, \quad$ whose inverse is $\quad r=\frac{\rho^{1 /(d-1)}}{(1-\rho G(\theta))^{1 /(d-1)}}$,

is a diffeomorphism from the region $\dot{\theta}>0$ into its image. If we transform equation (4) using the transformation (5), we obtain the following Abel equation

$$
\begin{aligned}
\frac{d \rho}{d \theta} & =-(d-1) G(\theta) F(\theta) \rho^{3}+\left[(d-1)\left(F(\theta)-G^{\prime}(\theta)\right] \rho^{2}\right. \\
& =A(\theta) \rho^{3}+B(\theta) \rho^{2}+C \rho .
\end{aligned}
$$

The solution $\rho\left(\theta, \rho_{0}\right)$ of $(6)$ satisfying that $\rho\left(0, \rho_{0}\right)=\rho_{0}$ can be expanded in a convergent series of $\rho_{0} \geq 0$ sufficiently small of the form

$$
\rho\left(\theta, \rho_{0}\right)=\rho_{1}(\theta) \rho_{0}+\rho_{2}(\theta) \rho_{0}^{2}+\rho_{3}(\theta) \rho_{0}^{3}+\cdots
$$

with $\rho_{1}(\theta)=1$ and $\rho_{k}(0)=0$ for $k \geq 2$. Let $P:\left[0, \tilde{\rho}_{0}\right] \rightarrow \mathbb{R}$ be the Poincaré return map defined by $P\left(\tilde{\rho}_{0}\right)=\rho\left(2 \pi, \tilde{\rho}_{0}\right)$ for a convenient $\tilde{\rho}_{0}$. System (1) has a center at the origin if and only if $\rho_{k}(2 \pi)=0$ for every $k \geq 0$. If we assume that $\rho_{2}(2 \pi)=\cdots=\rho_{m-1}(2 \pi)=0$ we say that $v_{m}=\rho_{m}(2 \pi)$ is the $m$-th Poincaré-Liapunov-Abel constant of system (1). Of course the set of coefficients for which all the PoincaréLiapunov-Abel constants $v_{m}$ vanish is the same that the set for which all the Poincaré-Liapunov constants $V_{2 k}$ vanish. This set, as we mentioned, is the center variety of system (1). 
We note that the space of systems (1) with a center at the origin is invariant with respect to the action group $\mathbb{C}^{*}$ of change of variables $z \rightarrow \xi z$ :

$$
\begin{array}{ll}
A \rightarrow \xi^{(d-7) / 2} \bar{\xi}^{(d-5) / 2} \xi^{5} A, & B \rightarrow \xi^{(d-7) / 2} \bar{\xi}^{(d-5) / 2} \xi^{4} \bar{\xi} B . \\
C \rightarrow \xi^{(d-7) / 2} \bar{\xi}^{(d-5) / 2} \xi^{3} \bar{\xi}^{2} C, & D \rightarrow \xi^{(d-7) / 2} \bar{\xi}^{(d-5) / 2} \xi^{2} \bar{\xi}^{3} D . \\
E \rightarrow \xi^{(d-7) / 2} \bar{\xi}^{(d-5) / 2} \xi \bar{\xi}^{4} E, & F \rightarrow \xi^{(d-7) / 2} \bar{\xi}^{(d-5) / 2} \bar{\xi}^{5} F,
\end{array}
$$

for a proof see [18].

The next result will be used to check when system (1) is reversible with respect to a straight line through the origin. Indeed system (1) is invariant with respect to a straight line through the origin if it is invariant under the change of variables $w=e^{i \gamma} z, \tau=-t$ for some real $\gamma$. The next result proved in [9].

Lemma 5. System (1) is reversible with respect to a straight line if and only if

$$
\begin{aligned}
& A=-\bar{A} e^{-4 i \gamma}, \quad B=-\bar{B} e^{-2 i \gamma}, \quad C=-\bar{C}, \\
& D=-\bar{D} e^{2 i \gamma}, \quad E=-\bar{E} e^{4 i \gamma}, \quad F=-\bar{F} e^{6 i \gamma},
\end{aligned}
$$

for some $\gamma \in \mathbb{R}$. Furthermore, in this situation the origin of system (1) has a center at the origin.

During the proof of Theorem 3 we will also consider equation (1) and its complex conjugated equation given by

(9) $\dot{\bar{z}}=-i \bar{z}+(z \bar{z})^{\frac{d-5}{2}}\left(\bar{A} \bar{z}^{5}+\bar{B} \bar{z}^{4} z+\bar{C} \bar{z}^{3} z^{2}+\bar{D} \bar{z}^{2} z^{3}+\bar{E} \bar{z} z^{4}+\bar{F} z^{5}\right)$.

We will also consider the complex system defined by both equations that after the complex change of time $t \rightarrow-i t$ is given by

$$
\dot{z}=z+P_{d}(z, \bar{z}), \quad \dot{\bar{z}}=-\bar{z}+Q_{d}(z, \bar{z}),
$$

where $P_{d}$ and $Q_{d}$ are homogeneous polynomials of degree $d$. Since we think there is no confusion we will also write it as

$$
\dot{x}=x+P_{d}(x, y), \quad \dot{y}=-y+Q_{d}(x, y) .
$$

We also present the following lemma given in [17] that we will need later in some cases.

Lemma 6. If system (11) has a local inverse integrating factor

$$
V=(x y)^{\alpha} \prod_{i=1}^{m} F_{i}^{\beta_{i}}
$$

with $F_{i}$ analytic in $x$ and $y, F_{i}(0,0) \neq 0$ for $i=1, \ldots, m, \alpha \neq 0$, and $\alpha$ not an integer greater than 1 , then it has an analytic first integral of the form $\Psi=x y+\cdots$. 
In fact this lemma is a particular case of Theorem 4.13 (iii) of [6].

\section{Proof of Theorem 1}

Proof of statement (a). The conditions of this case expressed in the real parameters are $a_{5}=a_{8}=0$ (i.e., $A=\operatorname{Re}(C)=D=0$ ) and

$$
\begin{aligned}
p_{1} & =a_{3} a_{9} a_{11}+a_{4} a_{10} a_{11}-a_{4} a_{9} a_{12}+a_{3} a_{10} a_{12}=0, \\
p_{2} & =a_{3}^{2} a_{9}-a_{4}^{2} a_{9}-2 a_{3} a_{4} a_{10}=0, \\
p_{3} & =a_{4} a_{9}^{2} a_{11}-3 a_{4} a_{10}^{2} a_{11}-a_{3} a_{9}^{2} a_{12}+4 a_{4} a_{9} a_{10} a_{12}-a_{3} a_{10}^{2} a_{12}=0, \\
p_{4} & =a_{4}^{2} a_{9} a_{11}+3 a_{3} a_{4} a_{10} a_{11}-a_{3} a_{4} a_{9} a_{12}+a_{3}^{2} a_{10} a_{12}=0, \\
p_{5} & =3 a_{3}^{2} a_{4} a_{11}-a_{4}^{3} a_{11}+a_{3}^{3} a_{12}-3 a_{3} a_{4}^{2} a_{12}=0, \\
p_{6} & =a_{9}^{3} a_{11}^{2}-3 a_{9} a_{10}^{2} a_{11}^{2}+6 a_{9}^{2} a_{10} a_{11} a_{12}-2 a_{10}^{3} a_{11} a_{12}-a_{9}^{3} a_{12}^{2}+3 a_{9} a_{10}^{2} a_{12}^{2} \\
& =0 .
\end{aligned}
$$

We now rewrite each of the conditions $p_{j}$ for $j=1, \ldots, 6$ in terms of complex parameters of system (1). We obtain that $p_{1}=\operatorname{Re}(\bar{B} E \bar{F})=0$ and $p_{2}=\operatorname{Re}\left(B^{2} E\right)=0$. Using that $p_{1}=0$ we get $p_{3}=\operatorname{Im}\left(B E^{2} \bar{F}\right)=0$, and using that $p_{1}=p_{2}=0$ we get $p_{4}=\operatorname{Im}\left(B^{2} E \bar{F}\right)=0$. Finally, we note that $p_{5}=\operatorname{Im}\left(B^{3} F\right)=0$ and $p_{6}=\operatorname{Re}\left(E^{3} \bar{F}^{2}\right)=0$. In summary, we have the conditions of statement (1).

From these conditions of statement (1) we have $A=D=0, \operatorname{Re}(C)=$ 0 that is $C=-\bar{C}$ and

$$
\frac{B}{\bar{B}}=-\frac{E \bar{F}}{\bar{E} F},\left(\frac{B}{\bar{B}}\right)^{2}=-\frac{\bar{E}}{E}, \frac{B}{\bar{B}}=\frac{\bar{E}^{2} F}{E^{2} \bar{F}},\left(\frac{B}{\bar{B}}\right)^{3}=\frac{\bar{F}}{F},\left(\frac{E}{\bar{E}}\right)^{3}=-\left(\frac{F}{\bar{F}}\right)^{2} .
$$

Now let $\theta_{1}, \theta_{2}, \theta_{3}$ be such that

$$
e^{i \theta_{1}}=-\frac{\bar{B}}{B}, \quad e^{i \theta_{2}}=-\frac{\bar{E}}{E}, \quad e^{i \theta_{3}}=-\frac{\bar{F}}{F} .
$$

From conditions (12) we have that

$$
\theta_{2}=-2 \theta_{1}(\bmod (2 \pi)), \theta_{3}=-3 \theta_{1}(\bmod (2 \pi)) .
$$

Now taking $\gamma=\theta_{1} / 2$ and using (13) we have

$$
\begin{aligned}
e^{2 i \gamma} & =e^{i \theta_{1}}=-\frac{\bar{B}}{B}, \quad e^{-4 i \gamma}=e^{-2 i \theta_{1}}=e^{i \theta_{2}}=-\frac{\bar{E}}{E}, \\
e^{-6 i \gamma} & =e^{-3 i \theta_{1}}=e^{i \theta_{3}}=-\frac{\bar{F}}{F} .
\end{aligned}
$$

Hence by Lemma 5 system (1) under the conditions of statement (1) is reversible and consequently has a center at the origin. 
Proof of statement (b). The conditions in the real parameters are $a_{5}=$ $a_{11}=a_{12}=3 a_{4}-a_{8}=a_{3}=0$. System (1) can be written into the form

$$
\begin{aligned}
\dot{z} & =i z+(z \bar{z})^{(d-5) / 2}\left(B z^{4} \bar{z}-3 \bar{B} z^{2} \bar{z}^{3}+E z \bar{z}^{4}\right) \\
& =i z+(z \bar{z})^{(d-3) / 2}\left(B z^{3}-3 \bar{B} z^{2}+E \bar{z}^{3}\right) .
\end{aligned}
$$

If we rescale system (14) by $|z|^{d-3}$ we get

$$
\dot{z}=i z|z|^{3-d}+B z^{3}-3 \bar{B} z^{2}+E \bar{z}^{3}=i \frac{\partial H}{\partial \bar{z}},
$$

where for $d=5$

$$
H=\log |z|^{2}-i\left(B z^{3} \bar{z}-\bar{B} z \bar{z}^{3}\right)-\frac{i}{4}\left(E \bar{z}^{4}-\bar{E} z^{4}\right),
$$

and for $d \geq 7$ odd we have

$$
H=\frac{2}{5-d}|z|^{5-d}-i\left(B z^{3} \bar{z}-\bar{B} z \bar{z}^{3}\right)-\frac{i}{4}\left(E \bar{z}^{4}-\bar{E} z^{4}\right) .
$$

Note that the first integrals $\exp (H)$ for $d=5$ and $H$ for $d \geq 7$ odd are real functions well defined at the origin. Therefore the origin is a center.

Proof of statement (c). The conditions in the real parameters are $a_{3}=$ $a_{5}=a_{9}=a_{11}=0$. Note that in this case we are under the assumptions of Lemma 5 with $\gamma=0$. Hence by Lemma 5 system (1) under the conditions of statement (3) is reversible and consequently has a center at the origin.

Proof of statement $(\mathrm{d})$. The conditions in the real parameters are $a_{5}=$ $a_{9}=a_{10}=2 a_{4}-a_{8}=a_{3}=0$. In this case system (1) takes the form

$$
\dot{z}=i z+(z \bar{z})^{(d-5) / 2}\left(B z^{4} \bar{z}-2 \bar{B} z^{2} \bar{z}^{3}+F \bar{z}^{5}\right) .
$$

Now we rescale by $(z \bar{z})^{(d-5) / 2}=|z|^{d-5}$ and system (15) becomes

$$
\dot{z}=i z|z|^{5-d}+B z^{4} \bar{z}-2 \bar{B} z^{2} \bar{z}^{3}+F \bar{z}^{5}=i \frac{\partial H}{\partial \bar{z}},
$$

where for $d \geq 5$ odd with $d \neq 7$ we have

$$
H=\frac{2}{7-d}|z|^{7-d}-\frac{i}{2} B z^{4} \bar{z}^{2}+\frac{i}{2} \bar{B} z^{2} \bar{z}^{4}-\frac{i}{6} F \bar{z}^{6}+\frac{i}{6} \bar{F} z^{6},
$$

and for $d=7$ we have

$$
H=\log |z|^{2}-\frac{i}{2} B z^{4} \bar{z}^{2}+\frac{i}{2} \bar{B} z^{2} \bar{z}^{4}-\frac{i}{6} F \bar{z}^{6}+\frac{i}{6} \bar{F} z^{6} .
$$

Note that the first integrals $\exp (H)$ for $d=7$ and $H$ for $d \geq 5$ odd with $d \neq 7$ are real functions well defined at the origin. Therefore in this case the origin is a Hamiltonian center. 


\section{Proof of Theorem 3}

Proof of statement (b). The conditions in the real parameters are $a_{3}=$ $a_{4}=a_{5}=a_{9}=5 a_{2}-a_{10}=0$. System (1) can be written into the form

$$
\dot{z}=i z+(z \bar{z})^{(d-5) / 2}\left(A z^{5}+i \operatorname{Im}(C) z^{3} \bar{z}^{2}-5 \bar{A} z \bar{z}^{4}+F \bar{z}^{5}\right) .
$$

If we rescale system (17) by $|z|^{d-5}$ we get

$$
\dot{z}=i z|z|^{5-d}+A z^{5}+i \operatorname{Im}(C) z^{3} \bar{z}^{2}-5 \bar{A} z \bar{z}^{4}+F \bar{z}^{5}=i \frac{\partial H}{\partial \bar{z}},
$$

where for $d \geq 5$ odd with $d \neq 7$ we have

$$
H=\frac{2}{7-d}|z|^{7-d}-i\left(A z^{5} \bar{z}-\bar{A} z \bar{z}^{5}\right)+\frac{\operatorname{Im}(C)}{3} z^{3} \bar{z}^{3}-\frac{i}{6}\left(F \bar{z}^{6}-\bar{F} z^{6}\right),
$$

and for $d=7$ we have

$$
H=\log |z|^{2}-i\left(A z^{5} \bar{z}-\bar{A} z \bar{z}^{5}\right)+\frac{\operatorname{Im}(C)}{3} z^{3} \bar{z}^{3}-\frac{i}{6}\left(F \bar{z}^{6}-\bar{F} z^{6}\right) .
$$

Note that the first integrals $\exp (H)$ for $d=7$ and $H$ for $d \geq 5$ odd, $d \neq 7$ are real functions well defined at the origin. Therefore the origin is a center.

Proof of statement (c). The conditions in real parameters are $a_{11}=$ $a_{12}=a_{9}=a_{5}=a_{2}+3 a_{10}=0$. In this case the associated complex differential system (11) is also a Lotka-Volterra case studied in [17]. In real coordinates system (1) under the conditions of this case becomes

$$
\begin{aligned}
\dot{x}=- & y+\left(x^{2}+y^{2}\right)^{(d-5) / 2}\left(a_{3} x^{5}+18 a_{10} x^{4} y-3 a_{4} x^{4} y-a_{6} x^{4} y\right. \\
& -2 a_{3} x^{3} y^{2}-28 a_{10} x^{2} y^{3}-2 a_{4} x^{2} y^{3}-2 a_{6} x^{2} y^{3} \\
& \left.-3 a_{3} x y^{4}+2 a_{10} y^{5}+a_{4} y^{5}-a_{6} y^{5}\right), \\
\dot{y}=x & -\left(x^{2}+y^{2}\right)^{(d-5) / 2}\left(2 a_{10} x^{5}-a_{4} x^{5}-a_{6} x^{5}-3 a_{3} x^{4} y\right. \\
& -28 a_{10} x^{3} y^{2}+2 a_{4} x^{3} y^{2}-2 a_{6} x^{3} y^{2}-2 a_{3} x^{2} y^{3} \\
& \left.+18 a_{10} x y^{4}+3 a_{4} x y^{4}-a_{6} x y^{4}+a_{3} y^{5}\right) .
\end{aligned}
$$

System (18) has the invariant curve $f=x^{2}+y^{2}$ and the inverse integrating factor $V=\left(x^{2}+y^{2}\right)^{(d+3) / 2}$ which, by integration, gives an analytic first integral at the origin.

Proof of statement $(\mathrm{d})$. The conditions in real parameters are $a_{11}=$ $a_{12}=a_{9}=a_{5}=a_{6}=a_{3}-a_{4}=7 a_{2}+a_{10}=49 a_{4}^{2}-8 a_{10}^{2}=0$. In this case the associated complex differential system (11) is also a LotkaVolterra case studied in [17]. We take $a_{3}=a_{4}$ and $a_{10}=-7 a_{2}$ and 
$a_{4}= \pm 2 \sqrt{2} a_{2}$. In this case the complex differential system (11) is given by

$$
\begin{aligned}
& \dot{x}=x+a_{2} x^{5} \pm(2-2 i) \sqrt{2} a_{2} x^{4} y-7 a_{2} x y^{4}, \\
& \dot{y}=-y+7 a_{2} x^{4} y \mp(2+2 i) \sqrt{2} a_{2} x y^{4}-a_{2} y^{5} .
\end{aligned}
$$

System (20) has the invariant curve of degree 8 given by

$$
\begin{aligned}
f(x, y) & =1+2 a_{2} x^{4}+a_{2}^{2} x^{8} \mp(2-2 i) \sqrt{2} a_{2} x^{3} y \mp\left(\frac{10}{3}-\frac{10 i}{3}\right) \sqrt{2} a_{2}^{2} x^{7} y \\
& -20 i a_{2}^{2} x^{6} y^{2} \mp(2+2 i) \sqrt{2} a_{2} x y^{3} \pm(18+18 i) \sqrt{2} a_{2}^{2} x^{5} y^{3} \\
& +2 a_{2} y^{4}-\frac{130}{3} a_{2}^{2} x^{4} y^{4} \pm(18-18 i) \sqrt{2} a_{2}^{2} x^{3} y^{5}+20 i a_{2}^{2} x^{2} y^{6} \\
& \mp\left(\frac{10}{3}+\frac{10 i}{3}\right) \sqrt{2} a_{2}^{2} x y^{7}+a_{2}^{2} y^{8} .
\end{aligned}
$$

Moreover, system (20) has the first integral $H(x, y)=x^{a} y^{b} f(x, y)^{c}$ where

$$
\begin{aligned}
a & =(-1)^{1 / 4}\left(3(-1)^{3 / 4}-(2-2 i) \sqrt{2}\right) / 3 \\
b & =i\left(3 i+(2+2 i)(-1)^{1 / 4} \sqrt{2}\right) / 3 \\
c & =-i\left(-3 i+(4+4 i)(-1)^{1 / 4} \sqrt{2}\right) / 6 .
\end{aligned}
$$

Proof of statement (e). The conditions in real parameters are $a_{11}=$ $a_{12}=a_{9}=a_{5}=a_{6}=a_{3}+a_{4}=7 a_{2}+a_{10}=49 a_{4}^{2}-8 a_{10}^{2}=0$. In this case the associated complex differential system 11 is also a LotkaVolterra case studied in [17]. We take $a_{3}=a_{4}$ and $a_{10}=-7 a_{2}$ and $a_{4}= \pm 2 \sqrt{2} a_{2}$. In this case the complex differential system (11) is given by

$$
\begin{aligned}
& \dot{x}=x+a_{2} x^{5} \pm(2+2 i) \sqrt{2} a_{2} x^{4} y-7 a_{2} x y^{4}, \\
& \dot{y}=-y+7 a_{2} x^{4} y \mp(2-2 i) \sqrt{2} a_{2} x y^{4}-a_{2} y^{5} .
\end{aligned}
$$

System (20) has the invariant curve of degree 8 given by

$$
\begin{aligned}
f(x, y) & =1+2 a_{2} x^{4}+a_{2}^{2} x^{8} \mp(2+2 i) \sqrt{2} a_{2} x^{3} y \mp\left(\frac{10}{3}+\frac{10 i}{3}\right) \sqrt{2} a_{2}^{2} x^{7} y \\
& -20 i a_{2}^{2} x^{6} y^{2} \mp(2-2 i) \sqrt{2} a_{2} x y^{3} \pm(18-18 i) \sqrt{2} a_{2}^{2} x^{5} y^{3} \\
& +2 a_{2} y^{4}-\frac{130}{3} a_{2}^{2} x^{4} y^{4} \pm(18+18 i) \sqrt{2} a_{2}^{2} x^{3} y^{5}+20 i a_{2}^{2} x^{2} y^{6} \\
& \mp\left(\frac{10}{3}-\frac{10 i}{3}\right) \sqrt{2} a_{2}^{2} x y^{7}+a_{2}^{2} y^{8} .
\end{aligned}
$$


Moreover, system (20) has the first integral $H(x, y)=x^{a} y^{b} f(x, y)^{c}$ where

$$
\begin{aligned}
a & =(-1)^{3 / 4}\left(3(-1)^{1 / 4}+(2+2 i) \sqrt{2}\right) / 3 \\
b & =\left(-3+(2+2 i)(-1)^{3 / 4} \sqrt{2}\right) / 3 \\
c & =\left(-3-(4+4 i)(-1)^{3 / 4} \sqrt{2}\right) / 6
\end{aligned}
$$

Proof of statement (f). The conditions in real parameters are $a_{11}=$ $a_{12}=a_{9}=a_{5}=a_{6}=a_{10}+3 a_{2}=0$ and $16 a_{2}^{2}-a_{3}^{2}-a_{4}^{2}=0$. In this case the associated complex differential system (10) is a Lotka-Volterra case studied in [17]. Doing the change $\xi=\left(1 / a_{2}\right)^{1 / 4}$ we can take $a_{2}=1$. Now taking $a_{3}= \pm 4 \cos \psi$ and $a_{4}= \pm 4 \sin \psi$, in real coordinates the system takes the form

$$
\begin{gathered}
\dot{x}=-y+4 x^{4} y+16 x^{2} y^{3}-4 y^{5} \pm 4 x^{5} \cos \psi \mp 8 x^{3} y^{2} \cos \psi \\
\mp 12 x y^{4} \cos \psi \mp 12 x^{4} y \sin \psi \mp 8 x^{2} y^{3} \sin \psi \pm 4 y^{5} \sin \psi, \\
\dot{y}=x+4 x^{5}-16 x^{3} y^{2}-4 x y^{4} \pm 12 x^{4} y \cos \psi \pm 8 x^{2} y^{3} \cos \psi \\
\mp 4 y^{5} \cos \psi \pm 4 x^{5} \sin \psi \mp 8 x^{3} y^{2} \sin \psi \mp 12 x y^{4} \sin \psi .
\end{gathered}
$$

In this case the complex differential system (11) is given by

$$
\begin{aligned}
& \dot{x}=x+x^{5}+3 x y^{4} \mp 4 i x^{4} y \cos \psi \pm 4 x^{4} y \sin \psi, \\
& \dot{y}=-y-3 x^{4} y-y^{5} \mp 4 i x y^{4} \cos \psi \pm 4 x y^{4} \sin \psi .
\end{aligned}
$$

System (22) is a Lotka-Volterra, consequently has the invariant curves $x=0, y=0$. Moreover it has the invariant curve of degree 12 given by $f=0$ where $f$ is

$$
\begin{aligned}
f & =1+24 x^{4} y^{4}\left(1+4 x^{4}+4 y^{4}\right) \\
& +4 x y\left[ \pm i(x-y)(x+y)\left(-3+4 x^{2} y^{2}\left(3+2\left(x^{2}-y^{2}\right)^{2}\right)\right) \cos \psi\right. \\
& -x y\left(9 y^{4}+x^{4}\left(9+16 y^{4}\right)\right) \cos 2 \psi-9 i x y\left(x^{4}-y^{4}\right) \sin 2 \psi \\
& \left. \pm\left(x^{2}+y^{2}\right)\left(3+4 x^{2} y^{2}\left(3+2\left(x^{2}+y^{2}\right)^{2}\right)\right) \sin \psi\right] .
\end{aligned}
$$

Moreover an inverse integrating factor of system (22) is given by $V=$ $x^{-1} y^{-1} f^{5 / 6}$. This inverse integrating factor is not well-defined at the origin. However applying Lemma 6 system (22) has an analytic first integral at the origin and consequently also system (21).

Proof of statement $(\mathrm{g})$. The four conditions of statement $(\mathrm{g})$ in real parameters are $a_{9}=a_{5}=a_{6}=a_{3}=a_{4}=3 a_{2}+5 a_{10}=16 a_{10}^{2}-$ $9\left(a_{11}^{2}+a_{12}^{2}\right)=0$. Doing the change of variables $\xi=\left(1 / a_{10}\right)^{1 / 4}$ and 
the last condition is $|F|=4\left|a_{10}\right| / 3$ we get that $F=4 / 3\left|a_{10}\right| e^{i \psi}$ with $\psi \in(0,2 \pi]$. Then we get

$$
\dot{z}=i z-i \frac{5}{3} z^{5}+i z \bar{z}^{4} \pm \frac{4}{3} e^{i \psi} \bar{z}^{5} .
$$

In real coordinates system (23) becomes

$$
\begin{aligned}
\dot{x}= & -y+\frac{34}{3} x^{4} y-\frac{44}{3} x^{2} y^{3}+\frac{2}{3} y^{5} \pm \frac{4}{3} x^{5} \cos \psi \mp \frac{40}{3} x^{3} y^{2} \cos \psi \\
& \pm \frac{20}{3} x y^{4} \cos \psi \pm \frac{20}{3} x^{4} y \sin \psi \mp \frac{40}{3} x^{2} y^{3} \sin \psi \pm \frac{4}{3} y^{5} \sin \psi, \\
\dot{y}= & x-\frac{2}{3} x^{5}+\frac{44}{3} x^{3} y^{2}-\frac{34}{3} x y^{4} \mp \frac{20}{3} x^{4} y \cos \psi \pm \frac{40}{3} x^{2} y^{3} \cos \psi \\
& \mp \frac{4}{3} y^{5} \cos \psi \pm \frac{4}{3} x^{5} \sin \psi \mp \frac{40}{3} x^{3} y^{2} \sin \psi \pm \frac{20}{3} x y^{4} \sin \psi .
\end{aligned}
$$

In this case the complex differential system (11) is given by

$$
\begin{aligned}
& \dot{x}=x-\frac{5}{3} x^{5}+x y^{4} \mp \frac{4}{3} i y^{5} \cos \psi \pm \frac{4}{3} y^{5} \sin \psi, \\
& \dot{y}=-y-x^{4} y+\frac{5}{3} y^{5} \mp \frac{4}{3} i x^{5} \cos \psi \mp \frac{4}{3} x^{5} \sin \psi .
\end{aligned}
$$

In fact if we compute some Poincaré-Liapunov constants for system (25) we obtain that the twelve first are zero but the next is not zero and its value is $V_{13}=\pi \sin 4 \psi$. Therefore we have that this constant only vanishes for $\psi=k \pi / 4$ with $k \in \mathbb{Z}$. Hence $\psi=0+k \pi, \psi=\pi / 2+k \pi$, $\psi=\pi / 4+k \pi$ and $\psi=3 \pi / 4+k \pi$ with $k \in \mathbb{Z} \backslash\{0\}$. The first two cases give time-reversible systems. For the third and fourth ones, system (25) takes the form

$$
\begin{aligned}
& \dot{x}=x-\frac{5}{3} x^{5}+x y^{4} \pm \frac{2 \sqrt{2}}{3}(1-i) y^{5}, \\
& \dot{y}=-y-x^{4} y+\frac{5}{3} y^{5} \mp \frac{2 \sqrt{2}}{3}(1+i) x^{5} .
\end{aligned}
$$

System (26) has not invariant algebraic curves of degree $\leq 16$ except the curve of fourth degree $f_{1}=1-x^{4} \pm(1-i) \sqrt{2} x^{3} y \pm(1+i) \sqrt{2} x y^{3}-y^{4}$. From now on we only work with the system (26) with upper signs to simplify the computations. For the other determination we can obtain similar results. We can write $f_{1}$ as $f_{1}=1-((-1-i) x+\sqrt{2} y)^{3}((1+i) x+$ $\sqrt{2} y) / 4$. This factorization suggests the following change of coordinates $X=(1+i) x+\sqrt{2} y$ and $Y=(-1-i) x+\sqrt{2} y$, whose inverse change is

$$
x=\frac{1}{4}(1-i)(X-Y), \quad Y=\frac{1}{2 \sqrt{2}}(X+Y) .
$$


In these new coordinates system (26) with the upper signs becomes

$$
\begin{aligned}
& \dot{X}=-Y+\frac{X^{5}}{16}+\frac{X^{3} Y^{2}}{2}+\frac{3 X Y^{4}}{16}, \\
& \dot{Y}=-X-\frac{X^{4} Y}{48}+\frac{X^{2} Y^{3}}{12}+\frac{Y^{5}}{48},
\end{aligned}
$$

and the invariant curve has the form $\tilde{f}_{1}=1-\left(X Y^{3}\right) / 4$. Now we made the transformation

$$
U=\frac{1-G / 12}{(1-G / 4)^{1 / 3}}-1, \quad V=-\frac{3 G^{2}+Y^{8}}{144(1-G / 4)^{2 / 3}},
$$

where $G=X Y^{3}$ and system (27) takes the form

$$
\dot{U}=V, \quad \dot{V}=-7(U+1) V-4\left(3 U+3 U^{2}+U^{3}\right) .
$$

Finally we made the rotation $U=u+v, V=-4 u-3 v$ and we obtain the system

$$
\begin{aligned}
& \dot{u}=-4 u-16 u^{2}+4 u^{3}-25 u v+12 u^{2} v-9 v^{2}+12 u v^{2}+4 v^{3} \\
& \dot{v}=-3 v+16 u^{2}-4 u^{3}+25 u v-12 u^{2} v+9 v^{2}-12 u v^{2}-4 v^{3}
\end{aligned}
$$

System (29) has a node at the origin whose eigenvalues are 3 and 4 and consequently is a linearizable node, see [7]. Moreover it is easy to check that going back through all the change of coordinates pulls back the meromorphic first integral (or the linearizing change of coordinates) to a first integral of the original system (26). So for this case we have a center.

Proof of statement $(\mathrm{h})$. The conditions in real parameters are $a_{3}=$ $a_{5}=a_{9}=a_{11}=0$. Note that in this case we are under the assumptions of Lemma 5 with $\gamma=0$. Hence by Lemma 5 system (1) under the conditions of statement (8) is reversible and consequently has a center at the origin.

Proof of statement (i). The conditions in real parameters are $a_{5}=a_{9}=$ $a_{10}=a_{4}-a_{12}=a_{3}+a_{11}=a_{2}-a_{6}=a_{6}^{2}-\left(a_{11}^{2}+a_{12}^{2}\right)$. Making the change of variables $\xi=\left(1 / a_{6}\right)^{1 / 4}$ and since the last condition is $|F|=|C|$ we get that $F=\left|a_{6}\right| e^{i \psi}$ with $\psi \in(0,2 \pi]$. Moreover we have $B=-\bar{F}$ that is, $B=-\left|a_{6}\right| e^{-i \psi}$. Then we get

$$
\dot{z}=i z+i z^{5} \mp e^{-i \psi} z^{4} \bar{z}+i z^{3} \bar{z}^{2} \pm e^{i \psi} \bar{z}^{5}
$$


In real coordinates system (30) becomes

$$
\begin{aligned}
\dot{x}= & -y-6 x^{4} y+8 x^{2} y^{3}-2 y^{5} \mp 8 x^{3} y^{2} \cos \psi \\
& \pm 8 x y^{4} \cos \psi \pm 2 x^{4} y \sin \psi \mp 12 x^{2} y^{3} \sin \psi+2 y^{5} \sin \psi, \\
\dot{y}= & x+2 x^{5}-8 x^{3} y^{2}+6 x y^{4} \mp x^{4} y \cos \psi \pm 8 x^{2} y^{3} \cos \psi \\
& \pm 2 x^{5} \sin \psi \mp 12 x^{3} y^{2} \sin \psi \pm 2 x y^{4} \sin \psi
\end{aligned}
$$

In this case the complex differential system (11) is given by

$$
\begin{aligned}
& \dot{x}=x+x^{5}+x^{3} y^{2} \pm i\left(x y^{4}-y^{5}\right) \cos \psi \pm\left(x^{4} y+y^{5}\right) \sin \psi, \\
& \dot{y}=-y-x^{2} y^{3}-y^{5} \mp i\left(x^{5}-x y^{4}\right) \cos \psi \mp\left(x^{5}+x y^{4}\right) \sin \psi
\end{aligned}
$$

System (32) has the invariant curve $f_{1}=1+\left(x^{2}+y^{2}\right)^{2}$ and the invariant curve of degree 8

$$
\begin{aligned}
f_{2}= & \frac{1}{4}\left(4+2\left(x^{2}+y^{2}\right)^{2}\left(2+3 x^{2} y^{2}\right)\right. \\
& +4 i x(x-y) y(x+y)\left(2+\left(x^{2}+y^{2}\right)^{2}\right) \cos \psi \\
& -\left(x^{2}+y^{2}\right)^{2}\left(x^{4}+y^{4}\right) \cos 2 \psi+4 x y\left(x^{2}+y^{2}\right)\left(2+\left(x^{2}+y^{2}\right)^{2}\right) \sin \psi \\
& \left.+i(x-y)(x+y)\left(x^{2}+y^{2}\right)^{3} \sin (2 \psi)\right)
\end{aligned}
$$

Moreover system (32) has an inverse integrating factor of the form $V=f_{1}^{1 / 4} f_{2}$ well-defined at the origin.

Proof of statement $(\mathrm{j})$. The conditions in real parameters are $a_{5}=a_{9}=$ $a_{10}=a_{4}+a_{12}=a_{3}-a_{11}=a_{2}+a_{6}=a_{6}^{2}-\left(a_{11}^{2}+a_{12}^{2}\right)$. Making the change of variables $\xi=\left(1 / a_{6}\right)^{1 / 4}$ and since the last condition is $|F|=|C|$ we get that $F=\left|a_{6}\right| e^{i \psi}$ with $\psi \in(0,2 \pi]$. Moreover we have $B=\bar{F}$ that is, $B=\left|a_{6}\right| e^{-i \psi}$. Then we get

$$
\dot{z}=i z-i z^{5} \pm e^{-i \psi} z^{4} \bar{z}+i z^{3} \bar{z}^{2} \pm e^{i \psi} \bar{z}^{5} .
$$

In real coordinates system (33) becomes

$$
\begin{aligned}
\dot{x}= & -y+4 x^{4} y-12 x^{2} y^{3} \pm 2 x^{5} \cos \psi \mp 12 x^{3} y^{2} \cos \psi \\
& \pm 2 x y^{4} \cos \psi \pm 8 x^{4} y \sin \psi \mp 8 x^{2} y^{3} \sin \psi, \\
\dot{y}= & x+12 x^{3} y^{2}-4 x y^{4} \mp 2 x^{4} y \cos \psi \pm 12 x^{2} y^{3} \cos \psi \\
& \mp 2 y^{5} \cos \psi \mp 8 x^{3} y^{2} \sin \psi \pm 8 x y^{4} \sin \psi .
\end{aligned}
$$

In this case the complex differential system (11) is given by

$$
\begin{aligned}
& \dot{x}=x-x^{5}+x^{3} y^{2} \mp i\left(x y^{4}+y^{5}\right) \cos \psi \mp\left(x^{4} y-y^{5}\right) \sin \psi, \\
& \dot{y}=-y-x^{2} y^{3}+y^{5} \mp i\left(x^{5}+x y^{4}\right) \cos \psi \mp\left(x^{5}-x y^{4}\right) \sin \psi
\end{aligned}
$$


System (35) has the invariant curve $f=1-\left(x^{2}+y^{2}\right)^{2}$ and the invariant curve of degree 8

$$
\begin{aligned}
f_{2}= & \frac{1}{4}\left(4-2\left(x^{2}-y^{2}\right)^{2}\left(2+3 x^{2} y^{2}\right)\right. \\
& +4 i x(x-y) y(x+y)\left(-2+\left(x^{2}-y^{2}\right)^{2}\right) \cos \psi \\
& +\left(x^{2}-y^{2}\right)^{2}\left(x^{4}+y^{4}\right) \cos 2 \psi-i\left(x^{2}-y^{2}\right)^{3} \sin 2 \psi \\
& +\left(x^{2}+y^{2}\right)\left(4 x y\left(-2+\left(x^{2}-y^{2}\right)^{2}\right) \sin \psi\right) .
\end{aligned}
$$

Moreover system (35) has an inverse integrating factor of the form $V=f_{1}^{1 / 4} f_{2}$ well-defined at the origin.

Proof of statement $(\mathrm{k})$. The conditions in real parameters are $a_{5}=$ $a_{9}=0$ and

$$
\begin{aligned}
& p_{1}=a_{2}-a_{10}=0 \\
& p_{2}=a_{4} a_{11}+a_{3} a_{12}=0, \\
& p_{3}=2 a_{6} a_{10}+a_{3} a_{11}-a_{4} a_{12}=0, \\
& p_{4}=a_{6}^{2}-a_{11}^{2}-a_{12}^{2}=0, \\
& p_{5}=a_{4} a_{6}-2 a_{10} a_{12}=0 \\
& p_{6}=a_{3} a_{6}+2 a_{10} a_{11}=0 \\
& p_{7}=a_{3}^{2}+a_{4}^{2}-4 a_{10}^{2}=0
\end{aligned}
$$

We can take $a_{6}=1$ by making the change $\xi=\left(1 / a_{6}\right)^{1 / 4}$. From $p_{1}=0$ we get $a_{2}=a_{10}$. Furthermore, condition $p_{4}=0$ implies $|F|=\left|a_{6}\right|$ and thus $F=\left|a_{6}\right| e^{i \psi}= \pm e^{i \psi}$, i.e., $a_{11}= \pm \sin \psi, a_{12}= \pm \cos \psi$. From $p_{5}=0$ we get $a_{4}=2 a_{10} a_{12}$ and from $p_{6}=0$ we get $a_{3}=-2 a_{10} a_{11}$. With these parameters we obtain that $p_{j}=0$ for $j=1, \ldots, 7$.

In real coordinates we get

$$
\begin{aligned}
\dot{x}= & -y-x^{4} y-2 a_{10} x^{4} y-2 x^{2} y^{3}+12 a_{10} x^{2} y^{3}-y^{5}-2 a_{10} y^{5} \\
& \pm 5 x^{4} y \cos \psi \mp 6 a_{10} x^{4} y \cos \psi \mp 10 x^{2} y^{3} \cos \psi \mp 4 a_{10} x^{2} y^{3} \cos \psi \\
& \pm y^{5} \cos \psi \pm 2 a_{10} y^{5} \cos \psi \pm x^{5} \sin \psi \mp 2 a_{10} x^{5} \sin \psi \\
& \mp 10 x^{3} y^{2} \sin \psi \pm 4 a_{10} x^{2} y^{3} \sin \psi \pm 5 x y^{4} \sin \psi \pm 6 a_{10} x y^{4} \sin \psi, \\
\dot{y}= & x+x^{5}+2 a_{10} x^{5}+2 x^{3} y^{2}-12 a_{10} x^{3} y^{2}+x y^{4}+2 a_{10} x y^{4} \\
& \pm x^{5} \cos \psi \pm 2 a_{10} x^{5} \cos \psi \mp 10 x^{3} y^{2} \cos \psi \mp 4 a_{10} x^{3} y^{2} \cos \psi \\
& \pm 5 x y^{4} \cos \psi \mp 6 a_{10} x y^{4} \cos \psi \mp 5 x^{4} y \sin \psi \mp 6 a_{10} x^{4} y \sin \psi \\
& \pm 10 x^{2} y^{3} \sin \psi \mp 4 a_{10} x^{2} y^{3} \sin \psi \mp y^{5} \sin \psi \pm 2 a_{10} y^{5} \sin \psi .
\end{aligned}
$$


We can write this system into the complex saddle form (11) as

$$
\begin{aligned}
\dot{x}= & x+a_{10} x^{5}+x^{3} y^{2}+a_{10} x y^{4} \mp i y^{5}(i \cos \psi+\sin \psi) \\
& \mp i a_{10} x^{4} y(2 i \cos \psi-2 \sin \psi), \\
\dot{y}= & -y-a_{10} x^{4} y-x^{2} y^{3}-a_{10} y^{5} \pm x^{5}(-\cos \psi-i \sin \psi) \\
& \pm 2 a_{10} x y^{4}(-\cos \psi+i \sin \psi) .
\end{aligned}
$$

System (37) is Darboux integrable because it has 3 invariant algebraic curves of fourth degree of the form $f_{i}(0,0) \neq 0$ and with these 3 curves it is possible to construct an integrating factor of system (37) of the form $V=f_{1} f_{2} f_{3}$ and consequently it has a complex center at the origin.

Proof of statement (1). The conditions in real parameters are $a_{5}=a_{9}=$ $a_{6}=a_{4}-a_{12}=a_{3}+a_{11}=a_{2}+a_{10}=4 a_{10}^{2}-\left(a_{11}^{2}+a_{12}^{2}\right)=0$.

Making the change of variables $\xi=\left(1 / a_{10}\right)^{1 / 4}$, and since the last condition is $|F|=2|E|$ we get that $F=2\left|a_{10}\right| e^{i \psi}$ with $\psi \in(0,2 \pi]$. Moreover we have $B=-\bar{F}$ that is, $B=-2\left|a_{10}\right| e^{-i \psi}$. Then we get

$$
\dot{z}=i z-i z^{5} \pm 2 e^{-i \psi} z^{4} \bar{z}+i z \bar{z}^{4} \pm 2 e^{i \psi} \bar{z}^{5} .
$$

In real coordinates system (38) becomes

$$
\begin{aligned}
\dot{x}= & -y+8 x^{4} y-8 x^{2} y^{3} \mp 16 x^{3} y^{2} \cos \psi \pm 16 x y^{4} \cos \psi \\
& \pm 4 x^{4} y \sin \psi \mp 24 x^{2} y^{3} \sin \psi \pm 4 y^{5} \sin \psi, \\
\dot{y}= & x+8 x^{3} y^{2}-8 x y^{4} \mp 16 x^{4} y \cos \psi \pm 16 x^{2} y^{3} \cos \psi \\
& \pm 4 x^{5} \sin \psi \mp 24 x^{3} y^{2} \sin \psi \pm 4 x y^{4} \sin \psi .
\end{aligned}
$$

We can write this system into the complex saddle form (11) as

$$
\begin{aligned}
& \dot{x}=x-x^{5}+x y^{4} \pm 2 i\left(x^{4} y-y^{5}\right) \cos \psi \pm 2\left(x^{4} y+y^{5}\right) \sin \psi, \\
& \dot{y}=-y-x^{4} y+y^{5} \mp 2 i\left(x^{5}-x y^{4}\right) \cos \psi \mp 2\left(x^{5}+x y^{4}\right) \sin \psi .
\end{aligned}
$$

System (40) is Darboux integrable because it has three invariant algebraic curve of degree 4 given by $f_{1}=1-\left(x^{2}+y^{2}\right)^{2}$ and two more curves that we do not write here due to their extension. In order to prove their existence we take polar coordinates $x=r \cos \theta, y=r \sin \theta$ in the real system (39) and following [12] we impose the existence of an invariant algebraic curve of degree $a$ that in polar coordinates takes the form $f=1+U_{1}(\theta) r^{4}$, i.e., $f$ must satisfies the equation

$$
\frac{\partial f}{\partial r} \dot{r}+\frac{\partial f}{\partial \psi} \dot{\psi}-\left(U^{\prime}(\psi) r^{4}\right) f \equiv 0
$$

Now substituting $U_{1}(\theta)$ by an arbitrary homogeneous polynomial of degree 4, i.e., taking $U_{1}(\theta)=B_{1} \cos 4 \theta+B_{2} \sin 4 \theta+B_{3} \cos 2 \theta+B_{4} \sin 2 \theta+$ 
$B_{5}$, it is easy to proof that equation (41) has three solutions $f_{1}, f_{2}$ and $f_{3}$ where $f_{1}$ has been given previously. Moreover $V=f_{1}^{-1 / 2} f_{2} f_{3}$ is an inverse integrating factor of system (40).

Proof of statement $(\mathrm{m})$. The conditions in real parameters are $a_{5}=$ $a_{9}=a_{6}=a_{4}+a_{12}=a_{3}-a_{11}=a_{2}+a_{10}=4 a_{10}^{2}-\left(a_{11}^{2}+a_{12}^{2}\right)=0$. Making the change of variables $\xi=\left(1 / a_{6}\right)^{1 / 4}$ and since the last condition is $|F|=2|E|$ we get that $F=2\left|a_{10}\right| e^{i \psi}$ with $\psi \in(0,2 \pi]$. Moreover we have $B=\bar{F}$ that is, $B=2\left|a_{10}\right| e^{-i \psi}$. Then we get

$$
\dot{z}=i z-i z^{5} \mp 2 e^{-i \psi} z^{4} \bar{z}+i z \bar{z}^{4} \pm 2 e^{i \psi} \bar{z}^{5} .
$$

In real coordinates system (42) becomes

$$
\begin{gathered}
\dot{x}=-y+8 x^{4} y-8 x^{2} y^{3} \pm 4 x^{5} \cos \psi \mp 24 x^{3} y^{2} \cos \psi \\
\quad \pm 4 x y^{4} \cos \psi \pm 16 x^{4} y \sin \psi \mp 16 x^{2} y^{3} \sin \psi, \\
\dot{y}=x+8 x^{3} y^{2}-8 x y^{4} \mp 4 x^{4} y \cos \psi \pm 24 x^{2} y^{3} \cos \psi \\
\mp 4 y^{5} \cos \psi \mp 16 x^{3} y^{2} \sin \psi \pm 16 x y^{4} \sin \psi .
\end{gathered}
$$

We can write this system into the complex saddle form (11) as

$$
\begin{aligned}
& \dot{x}=x-x^{5}+x y^{4} \mp 2 i\left(x^{4} y+y^{5}\right) \cos \psi \mp 2\left(x^{4} y-y^{5}\right) \sin \psi, \\
& \dot{y}=-y-x^{4} y+y^{5} \mp 2 i\left(x^{5}+x y^{4}\right) \cos \psi \mp 2\left(x^{5}-x y^{4}\right) \sin \psi .
\end{aligned}
$$

System (44) is Darboux integrable because it has three invariant algebraic curve of degree 4 given by $f_{1}=1-\left(x^{2}-y^{2}\right)^{2}$ and two more curves that we do not write here due to their extension. However as the previous case we can proof their existence. Moreover this case also has an inverse integrating factor of the form $V=f_{1}^{-1 / 2} f_{2} f_{3}$.

Proof of statement $(\mathrm{n})$. The conditions in real parameters are $a_{4}=$ $a_{5}=a_{9}=a_{12}=0$. Note that in this case we are under the assumptions of Lemma 5 with $\gamma=\pi / 2$. Hence by Lemma 5 system (1) under the conditions of statement (14) is reversible and consequently has a center at the origin.

\section{ACKNOWLEDGEMENTS}

We thank to Professor Colin Christopher for his help in the proof of statement $(\mathrm{g})$ of Theorem 3.

The first author is partially supported by a MINECO/ FEDER grant number MTM2011-22877 and an AGAUR (Generalitat de Catalunya) grant number 2014SGR 1204 . The second author is partially supported by a MINECO/ FEDER grant number MTM2008-03437, an AGAUR 
grant number 2009SGR 410, ICREA Academia, two FP7-PEOPLE2012-IRSES numbers 316338 and 318999, and FEDER-UNAB10-4E378. The third author is supported by Portuguese National Funds through FCT - Fundação para a Ciência e a Tecnologia within the project PTDC/MAT/117106/2010 and by CAMGSD.

\section{REFERENCES}

[1] N.N. BAUTIN, On the number of limit cycles which appear with the variation of coefficients from an equilibrium position of focus or center type, Mat. Sb. 30 (72) (1952), 181-196; Amer. Math. Soc. Transl. 100 (1954), 397-413.

[2] J. Chavarriga, H. Giacomini, J. Giné, J. Llibre, On the integrability of two-dimensional flows, J. Differential Equations 157 (1999), 163-182.

[3] J. Chavarriga, J. Giné, Integrability of a linear center perturbed by a fourth degree homogeneous polynomial, Publ. Mat. 40 (1996) 21-39.

[4] J. Chavarriga, J. Giné, Integrability of a linear center perturbed by a fifth degree homogeneous polynomial, Publ. Mat. 41 (1997) 335-356.

[5] L.A. Cherkas, The number of limit cycles of a certain second order autonumous system (Russian), Differencial'nye Uravnenija 12 (1976), no. 5, 944-946.

[6] C. Christopher, P. Mardešić, and C. Rousseau, Normalizable, integrable and linearizable saddle points for complex quadratic systems in $\mathbb{C}^{2}$, J. Dynam. Control Systems 9 (2003) 311-363.

[7] C. Christopher, C. Rousseau, Normalizable, integrable and linearizable saddle points in the Lotka-Volterra system, Qual. Theory Dynam. Syst. 5 (2004), no. 1, 11-61.

[8] W. Decker, S.Laplagne, G. Pfister, and H.A. Schonemann, SinGULAR 3-1 library for computing the prime decomposition and radical of ideals, primdec.lib, 2010.

[9] A. Cima, A. Gasull, V. Mañosa, F. Mañosas, Algebraic properties of the Liapunov and period constants, Rocky Mountain J. Math. 27 (1997), 471-501.

[10] P. Gianni, B. Trager, And G. Zacharias, Gröbner bases and primary decompositions of polynomials, J. Symbolic Comput. 6 (1988) 146-167.

[11] G.M. Greuel, G. Pfister, and H.A. Schönemann, Singular 3.0 A Computer Algebra System for Polynomial Computations, Centre for Computer Algebra, University of Kaiserlautern (2005). http: //www . singular . uni-kl. de.

[12] J. Giné, The center problem for a linear center perturbed by homogeneous polynomials, Acta Math. Sin. (Engl. Ser.) 22 (2006), no. 6, 1613-1620.

[13] J. Giné, On the centers of planar analytic differential systems, Internat. J. Bifur. Chaos Appl. Sci. Engrg. 17 (2007), 3061-3070.

[14] J. Giné, On the first integrals in the center problem, Bull. Sci. Math. 137 (2013), no. 4, 457-465.

[15] J. Giné, X. Santallusia, On the Poincaré-Lyapunov constants and the Poincaré series, Appl. Math. (Warsaw) 28 (2001), no. 1, 17-30.

[16] J. Giné, X. Santallusia, Implementation of a new algorithm of computation of the Poincaré-Liapunov constants, J. Comput. Appl. Math. 166 (2004), 465476. 
[17] J. Giné, V.G. Romanovski, Integrability conditions for Lotka-Volterra planar complex quintic systems, Nonlinear Anal. Real World Appl. 11 (2010) 2100-2105.

[18] J. LliBRE, C. VAlls, Classification of the centers and their cyclicity for the generalized quadratic polynomial differential systems, J. Math. Anal. Appl. 357 (2009), 427-437.

[19] J. Llibre, C. VAlls, Classification of the centers, their cyclicity and isochronicity for a class of polynomial differential systems generalizing the linear systems with cubic homogeneous nonlinearities, J. Differential Equations 246 (2009), 2192-2204.

[20] J. Llibre, C. VAlls, Classification of the centers and of the isochronous centers for a class of quartic-like systems, Nonlinear Analysis: Theory and Methods and Applications 71 (2009), 3119-3128.

[21] J. Llibre, C. VAlls, Classification of the centers, of their cyclicity and isochronicity for two classes of generalized quintic polynomial differential systems, NoDEA Nonlinear Differential Equations Appl. 16 (2009), no. 5, 657679.

[22] J. Llibre, C. VAlls, Centers and isochronous centers for two classes of generalized seventh and ninth systems, J. Dynamics and Differential Equations 22 (2010), 657-675.

[23] J. Llibre, C. VAlLs, Classification of the centers and their isochronicity for a class of polynomial differential systems of arbitrary degree, Advances in Mathematics 227 (2011), 472-493.

[24] M.A. Lyapunov, Problème général de la stabilité du mouvement, Ann. of Math. Stud. 17, Princeton University Press, 1947.

[25] H. PoIncaré, Sur l'intégration des équations différentielles du premier ordre et du premier degré I and II, Rendiconti del circolo matematico di Palermo 5 (1891), 161-191; 11 (1897), 193-239.

[26] V.G. Romanovski, M. PREŠERn, An approach to solving systems of polynomials via modular arithmetics with applications, J. Comput. Appl. Math. 236 (2011), no. 2, 196-208.

[27] D. Schlomiuk, Algebraic and geometric aspects of the theory of polynomial vector fields, in: Bifurcations and Periodic Orbits of Vector Fields, Montreal, PQ, 1992, in: NATO Adv. Sci. Inst. Ser. C Math. Phys. Sci., vol. 408, Kluwer Acad. Publ., Dordrecht, 1993, pp. 429-467.

[28] D. Schlomiuk, Algebraic particular integrals, integrability and the problem of the center, Trans. Amer. Math. Soc. 338 (1993) 799-841.

[29] K.S. SiBIRSKII, On the number of limit cycles in the neighborhood of a singular point (Russian) Differencial'nye Uravnenija 1 (1965) 53-66; Differential Equations 1 (1965) 36-47.

[30] N.I. VulPE, K.S. SiBIRSKII, Centro-affine invariant conditions for the existence of a center of a differential system with cubic nonlinearities (Russian) Dokl. Akad. Nauk SSSR 301 (1988), no. 6, 1297-1301; translation in Soviet Math. Dokl. 38 (1989), no. 1, 198-201.

[31] H. ŻOŁADEK, The classification of reversible cubic systems with center, Topol. Methods Nonlinear Anal. 4 (1994) 79-136. 
[32] H. ŻOĐADEK, Remarks on: The classification of reversible cubic systems with center [Topol. Methods Nonlinear Anal. 4 (1994) 79-136], Topol. Methods Nonlinear Anal. 8 (1996) 335-342.

1 Departament de Matemàtica, Universitat de Lleida, Avda. Jaume II, 69; 25001 Lleida, Catalonia, Spain

E-mail address: gine@matematica.udl.cat

2 Departament de Matemàtiques, Universitat Autònoma de Barcelona, 08193 Bellaterra, Barcelona, Catalonia, Spain

E-mail address: jllibre@mat.uab.cat

3 Departamento de Matemática, Instituto Superior Técnico, Av. Rovisco Pais 1049-001, Lisboa, Portugal

E-mail address: cvalls@math.ist.utl.pt 